\title{
Optimal Wing Configuration of a Tethered Satellite System in Free Molecular Flow
}

\author{
Andrew Dominic Santangelo* and Glen E. Johnsont \\ University of Michigan, Ann Arbor, Michigan 48109
}

\begin{abstract}
In this paper we present an analysis of the Tethered Satellite/Wing System in free molecular flow (at altitude of $142 \mathrm{~km}$ ) in planar motion subject to impulse movements. The analysis indicates that a wing system could provide stable flight over a wide range of conditions.
\end{abstract}

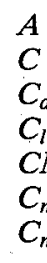

$A$
$C$
$C_{d}$
$C_{l}$
$C M$
$C_{m}$
$C_{m q}$

$D$
$D_{t}$
$d_{6}$
$I_{c m}^{0}, I$
$i$

$L$

$l$

$l_{\text {ref }}$

$M$
$M_{0 z}$

$R_{0}$

$S$

$S_{r}$

$T_{w}$

$t_{m}$

$v$

$w t$

$\alpha(t)$

$\alpha\left(t_{m}\right)$

$\alpha_{t m}\left(S, \alpha_{0}, d_{6}\right)$

$\alpha_{0}$
$\beta$

$\delta$

$\zeta$

$\rho$

$\omega_{n}$

\section{Nomenclature}

= Shuttle Orbiter altitude, $230 \mathrm{~km}$

$=$ aerodynamic damping derivative

$=$ drag coefficient, $2 D / \rho v^{2} S$

$=$ lift coefficient, $2 L / \rho v^{2} S$

$=$ center of mass

$=$ moment coefficient, $2 M / \rho v^{2} S l_{\text {ref }}$

$=$ pitch damping coefficient, $\partial(\mathrm{Cm}) /$

$\partial\left(\dot{\alpha} l_{\text {ref }} / v\right), 1 / \mathrm{rad}$

$=$ drag

= drag on satellite, $0.3 \mathrm{~N}$ (from Ref. 1)

$=$ boom length

$=$ moment of inertia of the TS/WS

$=$ inclination of the Orbiter plane relative to

the equator, $50 \mathrm{deg}$

$=$ lift

$=$ position along the tether measured from the Orbiter, $88 \mathrm{~km}$

= wing length

$=$ moment

$=$ moment resultant acting on the system

$=$ radius of the Earth, $6378.14 \mathrm{~km}$

$=$ wing area

$=$ speed ratio

$=$ wall temperature, $\approx 310 \mathrm{~K}$ (from Ref. 3 )

$=$ time of maximum angular overshoot

$=$ satellite speed

$=$ wing thickness, $0.048 \mathrm{~m}$

$=$ angle of attack

$=$ angular displacement of the TS/WS

$=$ maximum angular overshoot

$=\alpha\left(t_{m}\right)$ as a function of wing area, wing

inclination, and boom length

$=$ wing inclination

$=$ impulse magnitude factor

$=$ applied impulse moment

$=$ damping ratio of the TS/WS

$=$ density of the atmosphere, $3.358 \times 10^{-9}$

$\mathrm{kg} / \mathrm{m}^{3}$ (from Ref. 2)

$=$ natural frequency of the TS/WS
Received Jan. 31, 1991; revision received Sept. 18, 1991; accepted for publication Sept. 18, 1991. Copyright (C) 1992 by the American Institute of Aeronautics and Astronautics, Inc. All rights reserved.

*Graduate Research Assistant, Design Laboratory, Department of Mechanical Engineering and Applied Mechanics. Student Member AIAA.

†Associate Professor, Design Laboratory, Department of Mechanical Engineering and Applied Mechanics.

\section{Introduction}

$\mathbf{T}$ HE Tethered Satellite System (TSS), in conjunction with the Space Shuttle, will provide a new means for remote exploration of the Earth's upper atmosphere and ionosphere. To investigate the Earth's upper atmosphere, payloads of $200-500 \mathrm{~kg}$ will be lowered to distances of $100 \mathrm{~km}$ from the Orbiter (the TSS will investigate altitudes roughly between 130 and $220 \mathrm{~km}$ above the Earth)..$^{4-6}$ Stable flight is imperative for the success of the mission. One possible control strategy is the use of a flat plate passive wing system attached to a boom mounted on the TSS. Figure 1 shows a top view of the Tethered Satellite Wing System (TS/WS) as modeled in this study. The goal of this project was to find feasible and optimal values for wing area, boom length, and wing inclination to assure stable flight and acceptable peak "overshoot."

The model derived here is based on square flat plate wings. The TS/WS center of mass CM is located on the centerline of the boom, and the tether connection passes through the $C M$. The wing and boom dimensions are constrained by the practical fact that the system must "fit" within the Space Shuttle cargo bay. ${ }^{7}$
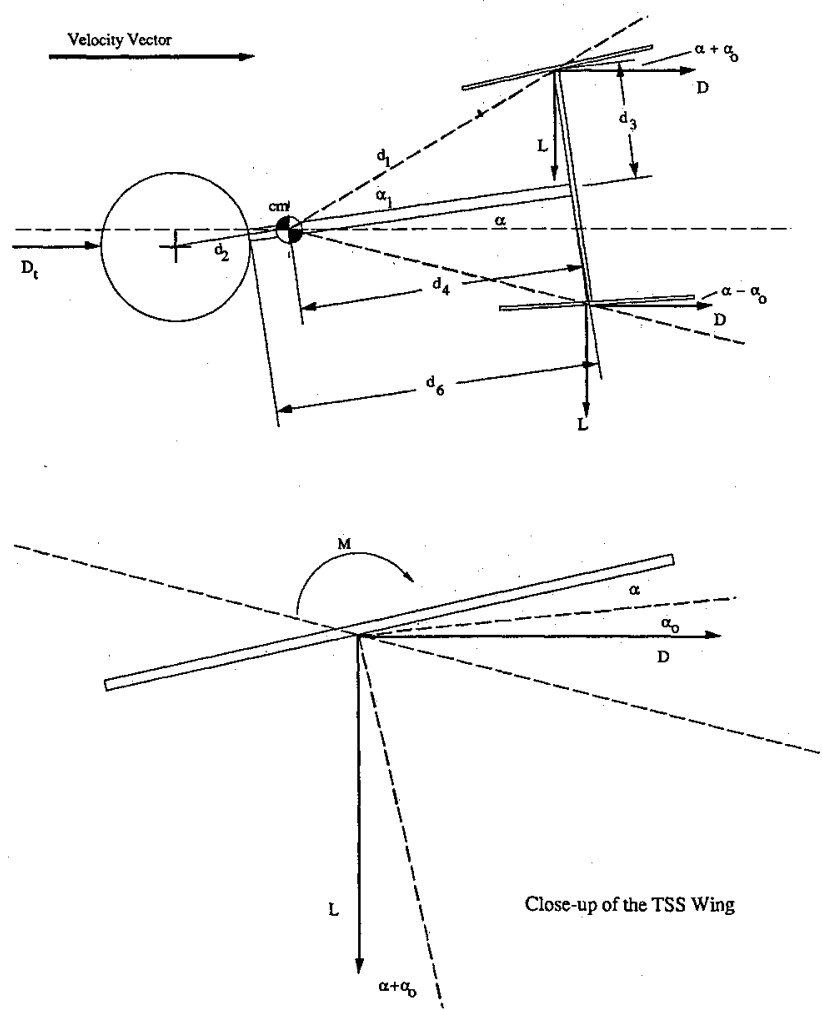

Fig. 1 Top view of the Tethered Satellite/Wing System with parameters and values used throughout the report. 
It is further assumed that the wings will remain in a fixed position, that the TS/WS is a symmetric body, and that the aluminum wing structure incorporates a protective coating to protect it from the hostile upper atmosphere and hypersonic velocities. This initial analysis is limited to planar motion.

\section{Background}

Although little work has been directed toward the problem of wing configuration optimization for the TSS, much related work has been done in the areas of system modeling, aerodynamics, and dynamics.

The conceptual design and mission requirements of the tethered satellite spherical model have already been posed. The TSS will be lowered to an altitude of $130 \mathrm{~km}$ where it will conduct its studies. Upon completion it will be further lowered until the tether breaks due to heating or is severed from the Shuttle. ${ }^{4,8}$ The satellite characteristics (the spherical portion) include a mass of $500 \mathrm{~kg}$ and a diameter of $1.25 \mathrm{~m} .^{1,9}$

The TSS satellite will fly in a very low pressure atmosphere where the Knudsen number is roughly 1-30 depending on the altitude. Since the TS/WS will fly in tandem with the Space Shuttle Orbiter at a speed of roughly $7400 \mathrm{~m} / \mathrm{s}$, the vehicle will travel at "hypersonic" velocities in a rarefied gas. In this range of Knudsen number, there are two regimes of flight that must be considered: transition flow $(\sim 100-140 \mathrm{~km})$ and free molecular flow $(>140 \mathrm{~km})$. This paper will examine flight in free molecular flow. NASA has studied the aerodynamic effects on the spherical part of the satellite and the connecting tether, including drag and aerodynamic heating ${ }^{1}$; also studied were conceptual models of a TSS wing system with a 45-deg half-angle cone frustrum attached to a 1-m-diam spherical satellite. ${ }^{3}$

In this article we examine a passive flat plate wing system. Aerodynamic models of the lift, drag, moment, and other coefficients for a flat plate in free molecular flow have been developed by Hayes and Probstein, ${ }^{10}$ Kogan, ${ }^{11}$ and Blick. ${ }^{12}$ Blick's model for flat plate aerodynamics was incorporated into the TS/WS simulation.

Optimization was completed through a parametric study with the computer application Mathematica. ${ }^{13}$

\section{Model Development}

Blick $^{12}$ analyzed and derived the aerodynamic coefficients as a function of angle of attack for a flat plate disk. Applying his result to the TSS flat plate wings leads to

$$
\begin{gathered}
C_{d}=2 \sin \alpha+\left(\sqrt{\pi} / S_{r}\right) \sin ^{2} \alpha \\
C_{l}=-\left(\sqrt{\pi} / S_{r}\right) \sin \alpha \cos \alpha \\
C_{m}=\left[-d_{4} \sqrt{\pi} \sin \alpha-2 S_{r} d_{4} \sin ^{2} \alpha\right. \\
\left.+2 S_{r} d_{3}(\cos \alpha)(\sin \alpha)\right] /\left(S_{r} l_{\mathrm{ref}}\right) \\
C_{m q}=\left[-S \sqrt{\pi}-12 d_{4}^{2} \sqrt{\pi}-(4 S) S_{r} \sin \alpha-24 S_{r} d_{3}^{2} \sin \alpha\right. \\
\left.-48 S_{r} d_{4}^{2} \sin \alpha+24 S_{r} d_{3} d_{4} \cos \alpha\right] /\left[(12 S) S_{r}\right]
\end{gathered}
$$

where $d_{3}$ and $d_{4}$ are defined in Fig. 1, and

$$
\begin{gathered}
S_{r}=v / \sqrt{2 R T_{w}} \\
v=\left(R_{0}+A-1\right)\left[\sqrt{\frac{G M}{\left(R_{0}+A\right)^{3}}}-\omega_{0} \cos i\right]
\end{gathered}
$$

For the specified environment, $S_{r}=17.74$ and $v=7376 \mathrm{~m} / \mathrm{s}$. Diffuse reflection with full surface accommodation was employed in the analysis, consistent with typical spacecraft surfaces.

Results for $C_{l}, C_{d}, C_{m q}$, and $C_{m}$ for one of the wings of the TS/WS as a function of $\alpha$ are shown in Fig. 2 .
The motion of a rigid body in a plane about a fixed point can be given by the following equation:

$$
M_{0 z}=I_{c m}^{0} \ddot{\alpha}
$$

where

$$
\begin{gathered}
M_{0 z}=\left(\frac{\partial M}{\partial \alpha}\right)_{\alpha=0} \alpha+\left(\frac{\partial M}{\partial \dot{\alpha}}\right)_{\dot{\alpha}=\alpha=0} \dot{\alpha}+\beta \delta \\
\left(\frac{\partial M}{\partial \alpha}\right)_{\alpha=0}=\frac{\partial C_{m}}{\partial \alpha}\left(0.5 \rho v^{2}\right) S l_{\mathrm{ref}}=-K \\
\left(\frac{\partial M}{\partial \dot{\alpha}}\right)_{\dot{\alpha}=\alpha=0}=C_{m q}\left(0.5 \rho v^{2}\right) S\left(l_{\mathrm{ref}} / v\right)=-C
\end{gathered}
$$

The $\beta \delta$ term is an applied impulse moment on the TS/WS, representing sudden flight environment changes. Applying the
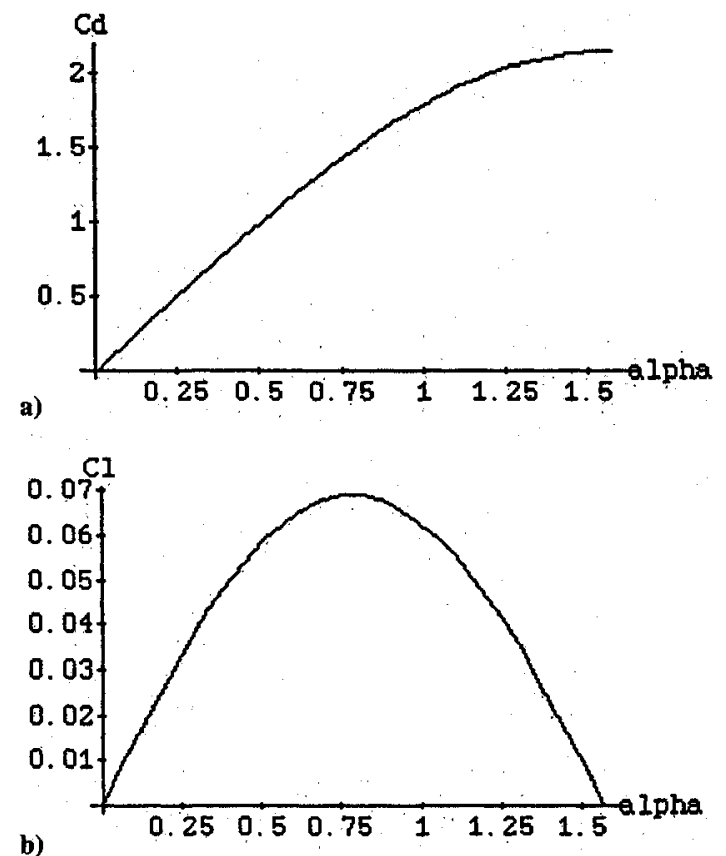

b)

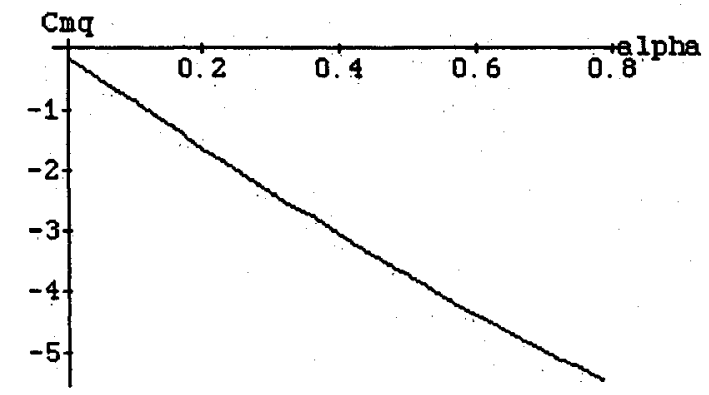

c)

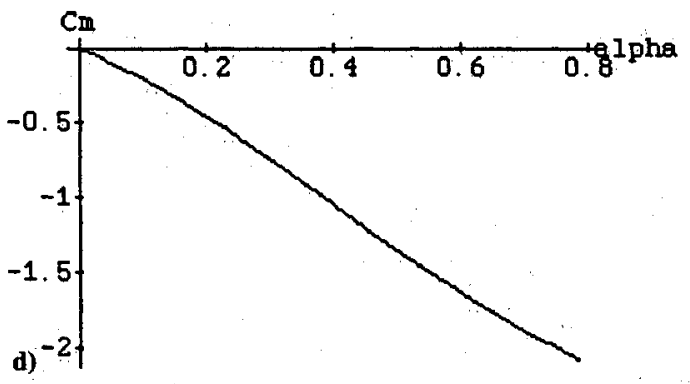

Fig. 2 Flat plate wing a) drag coefficient $\left(C_{d}\right)$, b) lift coefficient $\left(C_{l}\right)$, c) pitch damping coefficient $\left(C_{m q}\right)$, d) moment coefficient $\left(C_{m}\right)$ as a function of $\alpha$ (in rad). 
linearized equations (3) and (4), we get a differential equation in the form of

$$
I \ddot{\alpha}+C \dot{\alpha}+K \alpha=\beta \delta
$$

or

$$
\ddot{\alpha}+2 \zeta \omega_{n} \dot{\alpha}+\omega_{n}^{2} \alpha=\hat{F} \delta
$$

where

$$
\begin{gathered}
\zeta=\text { damping ratio }=C / 2 \sqrt{K I} \\
\omega_{n}=\text { natural frequency }=\sqrt{K / I} \\
\hat{F}=\beta / I
\end{gathered}
$$

and where $C, K$, and $I$ are dependent on the wing area $S$, the boom length $d_{6}$, and the wing inclination $\alpha_{0}$. Due to the rarefied environment, the resulting value of $C$ is much smaller than $K$.

The solution of the differential equation is

$$
\alpha(t)=\frac{\hat{F}}{\omega_{n} \sqrt{1-\zeta^{2}}} e^{-\zeta \omega_{n} t} \sin \omega_{n} \sqrt{1-\zeta^{2}} t
$$

The maximum overshoot $\alpha\left(t_{m}\right)$ of the system can be found by taking the derivative of the equation of motion and setting it equal to zero. Solving for $t_{m}$ yields

$$
t_{m}=\cos ^{-1} \zeta / \omega_{n} \sqrt{1-\zeta^{2}}
$$

Substitution of Eq. (16) into Eq. (15) gives

$$
\alpha\left(t_{m}\right)=\frac{\hat{F}}{\omega_{n} \sqrt{1-\zeta^{2}}} \exp \left(-\zeta \cos ^{-1} \zeta / \sqrt{1-\zeta^{2}}\right) \sin \left(\cos ^{-1} \zeta\right)
$$

which is a function of $S, d_{6}$, and $\alpha_{0}$. Equation (17) is the objective function to be minimized. The goal is to find values of $S, d_{6}$, and $\alpha_{0}$ that provide the minimum value for $\alpha\left(t_{m}\right)$.

The constraints are

$$
\begin{array}{ll}
g 1: & \alpha \leq \alpha_{\max }=2 \mathrm{deg} \\
g 2: & \alpha \geq \alpha_{\min }=-2 \mathrm{deg} \\
\text { g3: } & \alpha_{0} \leq \alpha_{0 \max }=45 \mathrm{deg} \\
\text { g4: } & \alpha_{0} \geq \alpha_{0 \min }=0 \mathrm{deg} \\
\text { g5: } & d_{6} \leq d_{6 \max }=6 \mathrm{~m} \\
\text { g6: } & d_{6} \geq d_{6 \min }=2 \mathrm{~m} \\
\text { g7: } & S \leq S_{\max }=5.3 \mathrm{~m}^{2} \\
\text { g8: } & S \geq S_{\min }=0.1 \mathrm{~m}^{2} \\
g 9: & C M \leq d_{6} \\
\text { g10: } & C M \geq C M_{\min }=0.2 \mathrm{~m} \\
\text { g11: } & \partial C_{m} / \partial \alpha<0
\end{array}
$$

A parametric study showed that $\alpha$ stays within the \pm 2 deg limits imposed by $g 1$ and $g 2$ for all feasible values of $S, d_{6}$, and $\alpha_{0}$. Hence $g 1$ and $g 2$ can be left out of the computation. Similarly, $g 9$ and $g 10$ (illustrated in Fig. 1) are never violated, so they can also be removed from the computation. From Fig. 2 one can see that the slope of the moment coefficient is negative, hence $g 11$ is also never violated. Obviously it would be prudent to test any "optimal" design to be sure that $g 1, g 2$, $g 9, g 10$, and $g 11$ are satisfied.
Table 1 Resultant peak overshoot due to an impulse moment, $\beta \delta$

\begin{tabular}{lc}
\hline$\beta$ & $\begin{array}{c}\text { Peak } \\
\text { overshoot, deg }\end{array}$ \\
\hline 0.5 & 0.1321 \\
1.0 & 0.2642 \\
2.0 & 0.5284 \\
4.0 & 1.056 \\
6.0 & 1.585 \\
8.0 & 2.113 \\
\hline
\end{tabular}

\section{Model Solution}

A parametric study indicated that the solution occurs at the maximum value for $d_{6}$. Accordingly, the boom length was fixed at $6 \mathrm{~m}$, and values for $S$ and $\alpha_{0}$ that would provide the minimum "'maximum peak overshoot" were determined. A parametric study of the objective function shows the optimal solution to occur at $S=5.3 \mathrm{~m}^{2}, \alpha_{0}=0 \mathrm{rad}$, and $d_{6}=6 \mathrm{~m}$. Numerical values of the optimal solutions for different values of the impulse magnitude factor $\beta$ are presented in Table 1. The results show that stable flight should occur for an impulse magnitude factor less than 7.6.

\section{Conclusion}

These results indicate that an appropriately configured passive wing system could provide stable flight for a TS/WS in free molecular flow subject to impulse moments. As a result, sensing probes on the satellite could accurately measure and study the Earth's upper atmosphere. The next step will be to investigate the behavior of the system under different loading conditions and transitional flow and in three-dimensional motion.

\section{Acknowledgment}

The authors of this paper wish to thank John D. Anderson for his help and guidance.

\section{References}

${ }^{1}$ Johnson, J. D., private communication, "Drag on the Proposed Tethered Satellites," Marshall Space Flight Center, Huntsville, AL, 1979.

${ }^{2}$ Anon., U.S. Standard Atmosphere, 1976, NASA, NOAA, and USAF, Washington, DC, 1976.

3Johnston, K. D., "Aerodynamics," Tethered Subsatellite Study, NASA TM X-73314, March 1976.

${ }^{4}$ Anderson, J. L., "Outer Atmospheric Research Using Tethered Systems," Journal of Spacecraft and Rockets, Vol. 26, No. 2, 1989, pp. 66-71.

5Anon., "Tethered Satellite System Facility Requirements," Center for Atmospheric and Space Sciences, Utah State Univ., Facility Requirements Definition Team Rept., Logan, UT, April 1980.

${ }^{6}$ Anon., Tethered Satellite System 2, NASA OAST, Washington, DC, May 1989.

${ }^{7}$ Kennedy, G. P., and Joels, K. M., The Space Shuttle Operator's Manual, Ballantine, New York, 1982.

${ }^{8}$ Anderson, J. L., Wood, G. M., and Siemers, P. M., "Research at the Earth's Edge," Aerospace America, Vol. 26, No. 4, April 1988.

${ }^{9}$ Anon., "Shuttle/Tethered Satellite System Conceptual Design Study," NASA TM X-73365, Dec. 1976.

${ }^{10}$ Hayes, W. D., and Probstein, R. F., Hypersonic Flow Theory, Vol. 5, 1st ed., Academic Press, New York, 1959, pp. 375-415.

${ }^{11}$ Kogan, M. N., Rarefied Gas Dynamics, Plenum Press, New York, 1969.

${ }^{12}$ Blick, E. F., "Forces on Bodies of Revolution in Free Molecular Flow by the Newtonian-Diffuse Method," McDonnell Douglas Aircraft Corp., Rept. No. 6670, St. Louis, MO, 1959.

${ }^{13}$ Wolfram, S., Mathematica, Addison-Wesley, Redwood City, CA, 1988.

Gerald T. Chrusciel Associate Editor 